\title{
Un mariage forcé ?
}

Les parents et l'école en France

A forced marriage? Parents and schools in France

¿Una boda forzada? Los padres y la escuela en Francia

\section{Judith Migeot-Alvarado}

\section{OpenEdition Journals}

Édition électronique

URL : https://journals.openedition.org/ries/1897

DOI : 10.4000/ries. 1897

ISSN : 2261-4265

\section{Éditeur}

France Education international

\section{Édition imprimée}

Date de publication : 1 décembre 2002

Pagination : $51-63$

ISBN : 978-2854-2-0555-8

ISSN : $1254-4590$

Référence électronique

Judith Migeot-Alvarado, « Un mariage forcé ? », Revue internationale d'éducation de Sèvres [En ligne], 31 | décembre 2002, mis en ligne le 12 avril 2012, consulté le 09 juillet 2021. URL : http:// journals.openedition.org/ries/1897 ; DOI : https://doi.org/10.4000/ries.1897 


\section{Un mariage forcé?}

\section{Les parents et l'école en France}

\section{Judith Migeot-Alvarado}

En France, lorsqu'on parle de participation parentale, on doit distinguer, d'une part, la démarche volontaire des parents d'élèves en tant que collectif, par le biais des associations, en vue d'une représentation au sein des instances décisionnaires à l'échelon national, régional ou local, et leur participation collective au fonctionnement des établissements scolaires; d'autre part, la démarche volontaire de participation individuelle aux travaux scolaires à la maison et la coopération individuelle à la vie de l'école ou de la classe.

Par famille, on doit comprendre aussi bien les parents, les beauxparents ou les parents adoptifs que d'autres membres de la famille, tels que les frères et sœurs plus âgés ou les grands-parents, ou tout autre membre de la famille qui s'implique dans l'école pour collaborer à l'éducation de l'enfant. Mais les familles ne forment pas un groupe homogène, elles n'éprouvent pas les mêmes besoins ni les mêmes attentes par rapport à l'école; elles n'ont pas non plus les mêmes styles éducatifs, ni les mêmes valeurs, ni les mêmes attitudes (Lautrey, 1980). Chaque famille, en fonction de sa position de classe, transmet un certain nombre d'atouts plus ou moins utiles pour réussir dans le milieu scolaire. On peut dire que ces atouts sont d'ordre économique, social et culturel (Lahire, 1995).

Depuis un quart de siècle, les formes de la vie familiale ont été profondément bouleversées en Europe. Les premiers signes de changement sont apparus dans les pays scandinaves, puis, progressivement, tous les pays du continent ont été concernés. Nuptialité en déclin rapide, augmentation considérable des taux de divorce, baisse rapide et importante du taux de natalité; avènement des formes de vie familiale hors mariage et augmentation des naissances hors mariage; proportion croissante des familles dites «monoparentales» ou encore réduction sensible de la taille moyenne des ménages. Ces changements concernent la plupart des pays européens, mais il subsiste pourtant des différences entre eux.

En France, la famille a aussi évolué. Outre les changements évoqués plus haut, la France est aussi confrontée au chômage des jeunes et à l'immigration. L'école, dans ces conditions, doit assurer non seulement une formation culturelle de base mais préparer également l'élève à sa future insertion professionnelle.

Nous avons choisi dans cet article de traiter les instances institutionnelles que la France a mises en place pour favoriser la participation des familles au fonctionnement des écoles. Mais nous sommes persuadée que l'analyse 
sociologique de leur participation ou de leur désaffection devrait compléter l'analyse institutionnelle. La participation des familles à l'école ne peut en effet s'appréhender indépendamment des biais sociaux et culturels affectant leur participation. Derrière l'apparente neutralité et égalité des instances institutionnelles dans leur vocation à accueillir les parents, percent encore fréquemment des disparités de modalités de participation suivant les différences de capital culturel et/ou social des familles (Henriot-van Zanten et MigeotAlvarado, 1992; 1995) ou suivant les domaines où les pouvoirs de l'État et des familles peuvent s'exercer, se concilier ou se combattre (Legrand, 1988) ou encore, suivant l'évolution historique des rapports entre l'école et la famille (Prost, 1981).

\section{POURQUOI FAIRE PARTICIPER LES PARENTS À L’ÉCOLE?}

Bien qu'enseignants et dirigeants soient d'accord pour dire que le soutien des parents aide les enfants à réussir à l'école, en France, malgré la récente décentralisation de l'administration, la participation des parents à l'école ne semble pas recueillir un large consensus de la part des enseignants.

La communication entre les parents et les enseignants est fortement institutionnalisée en France. En ce qui concerne le travail scolaire de l'élève, les parents sont informés par un bulletin trimestriel. Il est prévu également un cahier de liaison, ou cahier de correspondance, pour l'information au jour le jour, en cas de nécessité, entre les parents et les enseignants. Les parents sont tenus de le signer, et s'ils le souhaitent, de présenter leurs commentaires; ils peuvent également s'en servir pour demander un rendez-vous. Le mode de communication usuel entre la famille et l'école est le courrier, ce qui pose problème pour les familles qui ont des difficultés à parler la langue française ou des familles illettrées. C'est pourquoi dans certaines écoles, depuis quelques années, on met à la disposition des familles un médiateur ou traducteur pour venir en aide aux familles dans la relation avec l'école.

Nous avons vu que le souci de démocratisation s'est traduit par des efforts pour développer le rôle des familles à travers un certain nombre de mesures qui prévoient la participation des parents, et parfois des élèves, à la gestion des établissements. Les législations les plus récentes en France en ont souligné l'importance. Toutefois, la réponse des familles semble la plupart du temps décevante : les différentes fédérations de parents d'élèves se plaignent de la baisse de participation des parents aux élections.

Il y a toutefois un domaine pour lequel on constate un intérêt grandissant des parents. C'est celui de l'orientation. Le moment clé de l'orientation des élèves se situe au moment de l'articulation entre l'enseignement secondaire inférieur (collège) et l'enseignement secondaire supérieur général, technologique ou professionnel (lycées). C'est au niveau de la première classe de l'ensei- 
gnement secondaire supérieur, la seconde, que se trouve le plus grand nombre d'élèves redoublant, décrochant ou se réorientant : plus d'un sur quatre.

Des dispositions officielles ont aussi favorisé les évolutions dans ce sens. La loi française de 1989 précise que l'orientation doit se faire sur la base d'un accord entre l'élève, sa famille et l'établissement. Pour que le rôle des familles puisse se développer, il semblerait nécessaire qu'elles aient le sentiment de trouver la forme d'éducation répondant à leurs attentes. Selon A. Prost, la difficulté majeure rencontrée par la réforme de 1975 en France, est qu'elle a voulu offrir un enseignement de type secondaire alors que la demande sociale portait surtout sur un prolongement de l'enseignement primaire sans rupture avec les démarches traditionnelles de celui-ci (Prost, 1992).

\section{LA PARTICIPATION DES PARENTS AU FONCTIONNEMENT DES ÉTABLISSEMENTS ${ }^{1}$}

Malgré les dispositions officielles ${ }^{2}$, nos recherches montrent une autre image de la participation des parents à l'école. Les familles font preuve d'une grande méconnaissance du fonctionnement des établissements, surtout les familles des milieux populaires. Par ailleurs, très peu de parents connaissent le projet d'établissement du collège. L'insuffisance de l'information délivrée par les établissements est ressentie par les parents comme pouvant grever sérieusement le suivi scolaire de leurs enfants. Il faut sans doute voir là l'une des raisons de la désaffection des parents à l'égard des instances collectives de concertation.

En ce qui concerne les réunions de rentrée et les rencontres collectives par niveau, elles sont jugées positives, voire très positives, par un bon nombre des parents appartenant aux milieux favorisés, dans le sens qu'elles favorisent la rencontre avec les enseignants de la classe et avec l'équipe de direction. Néanmoins, un certain nombre de parents souhaite que le contenu de ces réunions soit modifié pour qu'y soient inclues des informations plus précises sur l'organisation pédagogique, l'orientation, les résultats aux examens, la vie des élèves dans l'établissement, et qu'y soient traités les problèmes extérieurs, tels que la sécurité, la drogue, etc.

En cours d'année, la principale réunion enseignants-parents est constituée par les rencontres individuelles où tous les professeurs reçoivent chaque famille. Ces rencontres semblent particulièrement investies par les parents des classes moyennes, et d'origine française, tandis qu'elles sont particulièrement

1. Cette partie reprend les grandes lignes d'une autre étude sur «La participation des parents au fonctionnement des établissements d'enseignement secondaire» menée avec A. Henriot-van Zanten, à la demande de la direction des lycées et collèges du ministère de l'Éducation nationale. Par des entretiens que nous avons conduits auprès des familles, nous avons cherché à connaître et à évaluer leurs pratiques individuelles et leur actions collectives susceptibles de favoriser ou de freiner les contacts avec l'administration et les enseignants.

2. Voir encadré p. 54. 


\section{Représentation des parents dans les instances de concertation et de décision}

\section{Au niveau national}

En France, les parents font l'objet de sollicitation de la part de l'administration depuis que certains textes leur donnent droit de cité au sein de l'institution scolaire. En effet, les deux dernières lois sur l'éducation (1975 et 1989) les désignent définitivement comme partenaires prioritaires des personnels d'enseignement et d'éducation, d'abord comme membres de la «communauté scolaire» puis de la «communauté éducative». Sept ans plus tard, dans une circulaire sur le «renforcement du dialogue entre les établissements et les parents d'élèves» (circulaire 96-117) le ministre insiste en soulignant «la nécessité de renforcer la coopération entre les parents d'élèves et les différents membres de la communauté scolaire, la reconnaissance et la valorisation du rôle des parents d'élève [...]. Des liens confiants entre les parents et les établissements scolaires constituent en effet un élément clé de la prévention de la violence à l'école et un fondement essentiel du projet éducatif de l'établissement». Plus récemment encore, la Semaine des parents à l'école (B.O. $\mathrm{n}^{\circ} 34$, septembre 98) est une action destinée «à instaurer un partenariat éducatif durable avec les parents dans un esprit d'ouverture, de confiance réciproque, de respect mutuel», et la Charte pour bâtir l'école $d u$ $X X I^{e}$ siècle (circulaire $\mathrm{n}^{\circ}$ 98-235) qui proclame l'ouverture de l'école, en particulier vers les parents, invités à participer et à collaborer.

Les parents sont associés à divers niveaux : au niveau national, ils sont représentés au Conseil supérieur de l'éducation (CSE). Les parents de l'école publique sont au nombre de neuf, ceux de l'école privée, trois.

\section{Au niveau des collectivités locales}

Les lois de décentralisation pour l'école de 1983 et de 1985 permettent aux collectivités de devenir une force de proposition en matière éducative. C'est ainsi que dans de nombreuses municipalités, les projets "pour la réussite de tous les enfants» se sont multipliés aux dernières élections municipales. Nombreuses sont également les équipes municipales qui proposent à l'ensemble de la population des "projets éducatifs» dont les principales actions se situent autour de l'aide au travail des enseignants, de l'accueil des enfants de deux ans à la maternelle, de la création des centres de ressources et d'aide à la lecture, de l'aménagement complet du temps de l'enfant, des échanges européens, de la création de clubs d'aide aux devoirs scolaires, parmi tant d'autres. En s'engageant massivement dans le domaine éducatif, les collectivités locales se posent de plus en plus comme partenaires exigeants de l'Éducation nationale, elles se sont saisies des pouvoirs nouveaux que leur confère la décentralisation et ont occupé un terrain jusqu'alors réservé à l'État. La politique des zones d'éducation prioritaire (ZEP), mise en œuvre dès 1982, dont le but était «de contribuer à corriger l'inégalité sociale par le renforcement sélectif de l'action éducative dans les zones et les milieux sociaux où le taux d'échec scolaire est le plus élevé» (circulaire de juillet 1981) a posé pour la première fois les problèmes éducatifs en termes géographiques (une zone comprend tous les établissements scolaires du territoire), et pour la première fois aussi, l'Éducation nationale faisait appel à ses partenaires extérieurs. Des modes de 
fonctionnement nouveaux ont alors vu le jour : commissions partenariales de zone réunissant tous les acteurs locaux (élus, enseignants, responsables d'établissements, travailleurs sociaux, représentants d'associations de parents d'élèves); groupe de pilotage en mairie avec l'élu chargé des affaires scolaires; nomination d'un coordinateur transversal, responsables des projets de l'ensemble des établissements de la ZEP, etc. En dehors des cadres habituels de l'Éducation nationale, des lieux de pouvoir et d'animation, sous tutelles diverses se sont mis en place, non sans difficulté. Enfin, le renforcement de la politique de développement social des quartiers et la création en 1988 de la délégation interministérielle de la ville, permettent d'aborder autrement les problèmes éducatifs. Toutefois les initiatives des villes se heurtent aux prérogatives des différents niveaux hiérarchiques de l'Éducation nationale. Dans les lois de décentralisation, l'État conserve le plein contrôle des orientations pédagogiques et du contenu des enseignements.

Au niveau des départements, les parents sont représentés au Conseil départemental tripartite de l'Éducation nationale (CDEN). Ils sont sept pour trente membres.

\section{Au niveau de l'établissement}

En France, les représentants élus des différentes associations, les parents délégués, sont officiellement légitimés pour participer aux différentes instances de concertation et de décision.

$\mathrm{Au}$ niveau de l'enseignement secondaire, les parents sont représentés dans plusieurs instances : au conseil d'administration des collèges et des lycées. La représentation des parents au conseil d'administration (CA) varie suivant le type et la taille de l'établissement, mais elle répond toujours au même principe de représentation tripartite entre le personnel, les usagers et l'administration. En général, dans un collège de moins de six cents élèves, six parents et deux élèves participent au CA (scrutin de liste pour les parents, élection par les délégués pour les élèves). Dans un lycée moyen, cinq parents et cinq élèves. Le conseil d'administration est l'organe décisionnel de l'établissement, il définit la politique de l'établissement dans le cadre prévu par la loi et fixe les principes de mise en œuvre de l'autonomie pédagogique et éducative. Les parents délégués participent également aux conseils de classe, où siègent les enseignants, le professeur principal de la classe, le chef d'établissement et/ou le conseiller principal d'éducation, le conseiller d'orientation et les délégués des élèves de la classe. Le conseil de classe se réunit au moins trois fois par an. Il examine le déroulement pédagogique de la classe, les notes des élèves ainsi que leurs comportements. Les propositions de redoublement et d'orientation sont du ressort du conseil de classe. En matière de redoublement ou d'orientation, le choix revient aux familles et aux élèves. En cas de désaccord entre la famille et l'établissement, la décision finale doit être précédée d'une rencontre avec la famille. Si la décision ne correspond pas aux souhaits des parents, ceux-ci peuvent faire appel de la décision du chef d'établissement auprès des autorités académiques. Le professeur principal a pour tâche de coordonner les observations des enseignants de la classe et d'être l'interlocuteur privilégié des familles sur les questions concernant le travail et les difficultés des élèves de la classe. Les parents sont représentés dans les commissions statuant en appel sur les questions d'orientation.

Au niveau du primaire, il n'existe pas de conseil de classe, mais de nombreux établissements ont un conseil d'établissement au sein duquel chaque classe est représentée par un parent. 
boudées par les familles populaires, et d'origine immigrée. Ce qu'il importe de noter, c'est qu'un consensus négatif se dégage à propos de ces rencontres, même chez les parents qui ne les fréquentent pas, et même chez ceux qui, par ailleurs, s'expriment difficilement. La critique porte essentiellement sur la manière dont ces réunions sont organisées : attente trop longue, entretiens trop courts et trop superficiels, sans aucune confidentialité.

Toutefois, un bon nombre de parents accorde une grande importance aux contacts individuels avec les enseignants, mais la fréquence de ceux-ci varie encore selon l'appartenance sociale des familles. Alors que la grande majorité des parents des classes aisées déclare avoir rencontré un ou plusieurs enseignants au moins une fois depuis le début de l'année, très peu de parents des classes modestes se trouvent dans ce cas. Les parents immigrés, quant à eux, sont ceux qui évitent le plus ce type de contact. Par ailleurs, on observe que les échanges enseignants-parents diminuent à mesure que le niveau d'enseignement des enfants monte. Les parents sont nombreux à regretter les différences sur ce point entre le collège et l'école primaire.

Au total, on observe que, majoritairement, les parents des établissements populaires ne peuvent être classés ni comme des «usagers " satisfaits, ni comme des «clients» exigeants. En effet, ils sont nombreux à se déclarer mécontents de la façon dont ils sont informés et des relations qu'ils entretiennent avec le personnel des établissements. Mais cette insatisfaction ne développe pas chez eux un désir de mobilisation collective : leur attitude semble être, au contraire, celle d'une relative résignation. On peut dire que l'implication des parents en tant que groupe dans les établissements scolaires est rendue difficile par le développement progressif d'un rapport individualiste des parents à l'école. Cependant, d'autres raisons concourent à expliquer cette situation. Nous en avons retenu deux qui nous semblent étroitement liées : la perception qu'ont les parents des instances de concertation et du rôle des parents délégués, et la perception qu'ils ont des associations de parents.

\section{Les parents délégués}

Pour ce qui est du rôle des instances de concertation (conseil de classe, conseil d'administration), il faut remarquer qu'il est mal connu par bon nombre de parents. Ces derniers estiment fréquemment que le conseil de classe est pour certains un moyen privilégié pour certains de recueillir des informations sur leur enfant et de multiplier les contacts avec les enseignants, ceci dans le but d'en retirer des bénéfices pour la scolarité de leurs propres enfants. Cette utilisation à des fins privées et intéressées des instances de concertation motive sans doute, pour une grande part, la méfiance des parents à l'égard de la délégation parentale et explique leur désimplication collective. Quant au rôle des parents délégués au conseil de classe, il est à noter que la grande majorité des familles ne connaît pas les parents délégués de la classe de leur enfant. Un petit 
nombre de parents estime leur rôle utile mais avec des réserves, alors qu'un bon nombre d'autres le juge totalement inutile.

Les parents qui ne contestent pas la nécessité du délégué parent considèrent toutefois que son activité réelle est doublement limitée : il est courtcircuité par l'action individuelle de nombreux parents et il est très peu écouté par l'administration et les enseignants (conseils de classes préparés à l'avance, imposition d'un temps de parole limité excluant tout débat...).

Les opinions franchement négatives se réfèrent à l'action de médiation du délégué parent. Elle apparaît comme étant inutile (dans la mesure où ce sont les parents qui doivent suivre directement la scolarité de leurs enfants et les enseignants qui doivent s'occuper de la progression des enfants) ou, plus fréquemment, comme étant humiliante (car cette médiation entraîne une plus grande visibilité de l'échec scolaire de certains enfants). En outre, certains parents pensent que toute forme de représentation est injuste et vouée à l'échec dans la mesure où les délégués plaident uniquement pour leur propre enfant oubliant ainsi leur engagement à l'égard de la collectivité des parents.

En ce qui concerne le rôle des parents délégués au conseil d'administration, presque tous les parents ignorent non seulement le but et le fonctionnement du conseil d'administration de l'établissement, mais aussi le rôle que doivent y jouer les parents délégués. Les parents qui sont mieux informés expriment une grande méfiance à l'égard des responsabilités effectivement attribuées aux parents, mais aussi parfois à l'égard de l'ensemble du dispositif.

\section{Les associations de parents}

Il s'avère que la grande majorité de parents n'adhère pas aux associations, à l'exception de "cadres et professions intellectuelles supérieures ». Très nombreux sont les parents qui émettent des critiques à l'égard des associations; elles sont de différentes sortes : certaines se réfèrent à l'impuissance des associations face aux décisions imposées au niveau national et face aux résistances des chefs d'établissement et des enseignants; d'autres concernent davantage le manque de représentativité des ces associations, d'autres encore évoquent la distance qui existe entre les représentants et les parents, d'autres enfin incriminent la dimension politique des associations.

Quant à l'idée d'un possible accroissement du pouvoir des associations, seulement un peu plus d'un quart des parents interrogés y est totalement favorable : ils souhaitent que les associations de parents soient en position de force pour débattre avec l'administration et avec les enseignants; ils souhaitent également que la bonne volonté des parents et leur compétence associative puissent être mise à profit au collège. De leur côté, les parents qui s'opposent à l'influence des associations, notamment les parents enseignants, évoquent le manque de compétence de la plupart des parents sur les questions pédagogiques, ce qui, à leur avis, limite inévitablement la capacité d’intervention des associations. 


\section{Réhabiliter la place des parents}

Pour conclure sur ce point, soulignons quelques observations qui risquent d'être de nature à mettre en péril le fonctionnement quotidien des établissements et par conséquent à porter atteinte à la poursuite même du projet de décentralisation et de démocratisation de l'école. L'absence d'intérêt et de participation effective des parents au fonctionnement des établissements semble tenir à plusieurs facteurs manifestes. En premier lieu, on observe chez la majorité des parents une grande méfiance à l'égard des structures de concertation : cela résulte d'un manque d'information sur leur fonctionnement, de la faible marge d'action qui leur est accordée par les textes officiels et de l'attitude des chefs d'établissements et des enseignants. Cependant, à supposer que ces structures retrouvent quelque crédit, deux écueils majeurs sont à éviter. Le premier est que si l'on continue à utiliser pour la transmission de l'information des supports qui sont principalement accessibles aux parents des milieux favorisés (documents écrits, réunions collectives...), on tendra à accroître les inégalités sociales devant l'école. Le second est que la diffusion des informations sur les résultats des enfants, ou certaines informations relatives à la gestion quotidienne de la vie scolaire, risque d'accroître la concurrence entre les établissements, laquelle est déjà particulièrement aiguë entre certains quartiers, et risque d'accroître la stigmatisation des établissements populaires. Cependant, un travail de sensibilisation est sans doute à faire auprès de l'administration et des enseignants pour tenter de réhabiliter la place des parents dans les établissements et la nécessité de les y intégrer, ceci dans le but de favoriser la réussite scolaire des enfants. Mais les dispositions des textes officiels n'y suffiront pas, il serait donc souhaitable d'inclure ou de développer ce travail de sensibilisation dans la formation continue du personnel enseignant et non enseignant, et dans la formation initiale aux métiers de l'enseignement. Il est probable que la participation des parents contribue à améliorer le fonctionnement des établissements locaux d'enseignement mais il faudrait éviter, dans la mesure du possible, les effets pervers que cet accueil pourrait entraîner, à savoir une plus grande visibilité des parents en difficulté, une stigmatisation de leurs difficultés à assumer l'éducation de leurs enfants, et par conséquent une évaluation morale de ces famille par l'école (Henriot-van Zanten, Migeot-Alvarado, 1992; 1995).

\section{RAPPORT AU SAVOIR, TRAVAIL SCOLAIRE ET IMPLICATION DES ENSEIGNANTS ET DES PARENTS}

Par ailleurs, il nous semble impossible de conclure cette analyse sans évoquer une autre étude ${ }^{3}$ qui fait état d'une autre série de facteurs, peut-être

3. Il s'agit d'une étude sur les parcours pédagogique diversifiés dans un certain nombre de collèges de l'académie de Besançon. Cette étude s'inscrit dans le cadre d'une recherche coordonnée par l'INRP. 
plus latents, et qui doivent être pris en compte dans toute perspective de remédiation. Il s'agit de poser maintenant la problématique du rapport au savoir et du travail scolaire dans le collège d'aujourd'hui. Comment parents, enfants et éducateurs peuvent-il ouvrer ensemble dans un projet éducatif commun sans que les parents s'immiscent dans le travail pédagogique des enseignants, sans que les enseignants empiètent sur la vie privée des parents et sans que les enfants se sentent tiraillés entre les deux? Certains travaux montrent que des «réussites paradoxales» existent par le fait que certains élèves dont les caractéristiques sociales et culturelles prédisposent à l'échec scolaire, connaissent des réussites honorables voir brillantes. Ces réussites semblent résulter du rapport au savoir des élèves et de leur mobilisation à l'école et sur l'école. Les élèves qui découvrent le plaisir d'apprendre, qui donnent au travail scolaire un sens proprement cognitif ( «on apprend pour connaître» et non pas pour des motivations utilitaristes) réussissent mieux à l'école quelle que soit leur origine sociale et ethnique (Charlot, Bautier et Rochex, 1992).

\section{Droits et représentations des élèves}

En France, comme dans d'autres pays de l'Union européenne, l'enfant-élève est considéré comme un être autonome disposant de prérogatives pouvant se conjuguer avec celles qui sont reconnues aux parents, mais aussi de droits propres. Ces droits propres s'entendent tout d'abord comme garanties procédurales (Fialaire, 1996). En France, par exemple, le décret du 18 décembre 1985 fixant la procédure disciplinaire prévoit que lors de la tenue d'un conseil de discipline, le chef d'établissement est chargé de convoquer, outre l'élève et son représentant légal lorsque ce dernier est mineur, une personne éventuellement chargée d'assister l'élève pour présenter sa défense. Les garanties des droits des élèves ont été renforcées en France par la loi d'orientation sur l'éducation (1989), complétée par les différents décrets d'application, parmi lesquels le décret de 1991 (relatif aux droits et obligations des élèves des établissements publics locaux d'enseignement, reconnaissant à ces élèves différents fondamentaux rattachés à la personne humaine, dont la liberté d'opinion dans certaines limites, la liberté de conscience et le droit au respect de l'intégrité physique) et le décret de 1990 établissant une procédure d'appel des décisions d'orientation des élèves garantissant la possibilité d'une audition des parents d'élèves.

La participation des élèves est considérée comme faisant partie de la formation à la démocratie et à la citoyenneté, c'est pourquoi les élèves disposent de conseils d'élèves, et c'est pourquoi ils sont représentés au sein des instances administratives scolaires. En France, les délégués sont élus à raison de deux par classe, qui participent au conseil de classe. Ils se réunissent pour désigner leurs représentants au conseil d'administration, à la commission permanente qui en émane, au conseil de discipline. Leur compétence est d'ordre consultatif. Les délégués des élèves reçoivent en principe une formation, souvent confiée aux conseillers d'éducation. 
Il y a sans doute là matière à réflexion. Si le collège trop souvent ne sait plus susciter le désir d'apprendre chez les élèves et s'il perd une partie de sa crédibilité chez certains parents, c'est qu'il a tendance à substituer à une relation de désir face au savoir une relation instrumentale. Cette instrumentalisation, loin de favoriser l'autonomie de l'élève dans l'«apprendre à apprendre", le rend dépendant vis-à-vis du soutien scolaire de parents qui bénéficient d'un capital scolaire, et accentue la fonction sélective de l'école. C'est pourquoi aujourd'hui, le collège est appelé de plus en plus à se légitimer devant les familles les moins favorisées culturellement et devant leurs enfants. Sans entrer dans un rapport de force, le collège peut se légitimer en montrant que le savoir a du sens, que l'étude peut aider l'enfant à éprouver la joie d'apprendre et de comprendre tout en devenant autonome, et en montrant que la présence de l'élève dans l'institution prend ainsi du sens.

\section{MOdifIER L'IMAge QUE LES ENSEIGNANTS ET LES FAMILLES ONT LES UNS DES AUTRES}

Le peu d'adhésion des familles aux structures de concertation, la distance critique ou indifférente qu'elles maintiennent à leur égard s'expliquent sans doute par le fait que les parents, qui se sentent exclus des lieux de décision, ou peu concernés par eux, comme nous venons de le voir, se sentent dans le même temps - et contradictoirement - convoqués, tacitement requis, par cette même école pour prolonger à la maison le travail des enseignants. Dans nos entretiens, nombreux sont les parents qui se plaignent de l'importance croissante que prend l'aide aux devoirs dans la vie familiale. Les échanges entre ces parents et leurs enfants sont très centrés sur les questions relatives au travail scolaire. L'enfant est perçu de plus en plus par la famille comme élève, ce qui produit des tensions à l'intérieur de la famille et des tensions entre la famille et l'institution scolaire. D'autant plus que les collégiens entrant dans l'adolescence vivent, selon beaucoup de parents, une période propice aux conflits familiaux qui trouvent naturellement à s'exprimer sur le terrain de la scolarité.

Une enquête récente révèle que les élèves qui obtiennent les meilleurs résultats sont ceux dont la mère est elle-même enseignante. La connaissance détaillée du système scolaire semble être en France un grand atout. La question des rapports entre les parents et l'école s'inscrit ainsi dans une problématique plus large, qui est celle du rôle de la scolarité dans le devenir social et professionnel des jeunes (OCDE, 1997).

Face aux problèmes des inégalités sociales dans le domaine de la réussite scolaire, on ne peut aujourd'hui continuer à chercher unilatéralement un bouc émissaire, soit du côté de l'établissement, soit du côté des familles. Du côté de l'école, on dit souvent qu'il suffirait d'introduire un changement dans l'organisation des savoirs, des méthodes et des pratiques pédagogiques pour lutter 
efficacement contre la production de ces inégalités, qu'il suffirait de mobiliser les familles pour que les enfants réussissent. Du côté des familles, on dit qu'il suffirait d'accroître les aides familiales et le soutien scolaire pour favoriser la réussite scolaire des enfants. S’il est vrai que les chances de réussite scolaire peuvent dépendre en grande partie de l'articulation de ces tactiques, il ne faut pas perdre de vue que leur conjonction ne saurait être efficace sans une politique économique de lutte contre le chômage et l'exclusion. En effet, les parents en situation de précarité extrême ne sont pas en état de soutenir - fût-ce moralement - le travail scolaire des enfants. Privés de toute reconnaissance sociale, ils ne sont plus en mesure d'affirmer le maintien d'exigences, et, d'autre part, ils offrent à leurs enfants le spectacle d'une réalité sociale qui ne peut qu'être de nature à désamorcer leur motivation et discréditer l'idée d'un «salut par l'école». Il ne s'agit nullement de dédouaner l'école de sa responsabilité, mais comme le dit Joshua de "ne pas se tromper de diagnostic» (Joshua, 1999) et imputer à l'institution scolaire des torts qui la dépassent et dont elle est la première victime.

Il reste au collège une voie praticable pour faire face aux difficultés : l'articulation entre l'éducatif et le pédagogique. En France, malgré le fait qu'un des objectifs de la création des instituts universitaires de formation des maîtres (IUFM) en 1990, était d'améliorer la formation initiale des enseignants du secondaire, cette formation demeure presque exclusivement centrée sur les connaissances disciplinaires; c'est pourquoi tout ce qui concerne les approches de l'éducation relevant de la psychologie, de la sociologie, de la philosophie, de l'histoire du système éducatif, qui peuvent donner aux futurs enseignants une meilleure compréhension des forces sociales, des comportements sociaux, des problèmes d'apprentissage de l'élève, sont en général étrangères à la formation initiale des professeurs, ou dans le meilleur de cas, elles ne restent qu'un supplément d'âme. Pourtant, certains pédagogues, en essayant d'agir au niveau de l'environnement scolaire et à l'intérieur même de la classe, développent de plus en plus des actions qui visent cette articulation : ils accompagnent la transmission du savoir de la formation aux pré-requis (institutionnels, culturels) sans lesquels ces savoirs restent lettre morte. De telles actions ne déboucheront pas seulement sur plus d'efficacité de l'acte pédagogique, mais assureront davantage d'équité au sein même de l'établissement. C'est dans ces conditions que le collège peut être un facteur d'égalité des chances. Ainsi, au lieu de jouer sur des complicités culturelles à travers des pédagogies implicites, l'institution s'obligera elle-même à dispenser une instruction totalement explicite, veillant à ne rien laisser au hasard des différences de fortune ou d'environnement culturel.

Nous avons vu qu'en France, d'un point de vue politique, l'instauration d'un véritable partenariat entre la famille et l'école est actuellement une question importante. Les pouvoirs publics ont pris beaucoup d'initiatives, notamment ces dernières années, pour que les parents soient considérés comme 
membres à part entière de la communauté éducative. Mais il est évident que la législation ne suffit pas et que, pour devenir réalité, ce partenariat doit passer sans doute par les initiatives locales. Les parents et les écoles ne sont pas en France des partenaires «naturels». On voit au contraire entre eux beaucoup d'incompréhensions, de différences de «culture» et de divergences d'intérêt. Mais la nécessité de placer les élèves au centre du système (loi d'orientation, 1989) et de faire en sorte que les parents les soutiennent, à l'école et avec l'école, force progressivement les enseignants et les familles à modifier l'image qu'ils ont les uns des autres. Le défi est désormais moins politique et plus social.

\section{Bibliographie}

AUBERT et al. (1985) : La forteresse enseignante : la Fédération de l'Éducation nationale, Paris, Fayard.

AUDUC J.L. (1994) : Le système éducatif, Paris, Hachette-Éducation.

BALLION R. (1982) : Les consommateurs d'école, Paris, Stock.

BOUNOURE A. (1995): Parents des collégiens. Conceptions et pratiques éducatives, Paris, INRP.

BOURDIEU P., CHAMPAGNE P. (1993): «Les exclus de l'intérieur» in BOURDIEU P. (dir.) La misère du monde, Paris, Le Seuil.

BOUVEAU P., COUSIN O., FAVRE J. (1999): L'école face aux parents. Analyse d'une pratique de médiation, Paris, ESF.

CE, Commission Européenne (1995): Le rôle des parents dans les systèmes éducatifs de l'Union européenne, Bruxelles, Eurydice.

DEROUET J.-L. (1988): «La profession enseignante comme montage composite» in Éducation permanente, Paris, $\mathrm{n}^{\circ} 96$.

DESCHAMPS J.-C. et al. (1982): L'échec scolaire : Élève modèle ou modèles d'élèves?, Lausanne, P.M. Favre.

DUBET F. et al. (1997) : École - familles : le malentendu, Paris, Textuel.

DURU-BELLAT M., VAN ZANTEN A. (1999): Sociologie de l'école, Paris, A. Colin ( $2^{\circ}$ éd.).

DUTERCQ Y. (1992): Consommateurs d'école et modernisation du service public, Paris, INRP, Groupe d'études sociologiques.

HENRIOT-VAN ZANTEN A., MIGEOT-ALVARADO J. (1992) : La participation des familles au fonctionnement des établissements d'enseignement secondaire. Rapport à la direction des lycées et des collèges du Ministère de l'Éducation nationale, Paris, Équipe de sociologie de l'éducation, Paris V.

HENRIOT-VAN ZANTEN A., MIGEOT-ALVARADO J. (1995): «Socialisation familiale et investissement scolaire. Au croisement d'une sociologie de la famille et d'une sociologie de l'école», L'Année sociologique, vol. 45 (1), 182-191. 
LAHIRE B. (1995): Tableaux des familles, Paris, Gallimard / EHESS.

LAUTREY (1980): Classe sociale, milieu familial, intelligence, Paris, PUF. MIGEOT-ALVARADO J. (1994): Los sistemas educativos en la Union Europea : Programas de accion en materia de Educacion, formación y empleo. Caracas, Cinterplan, O.E.A.

MIGEOT-ALVARADO J. (1997): Papel de los padres en la administración y gestion de las escuelas en la Union europea, Document de travail. Caracas, Cinterplan, O.E.A.

MIGEOT-ALVARADO J., La relation école-famille : Peut mieux faire, Paris ESF 2000.

MENRT, Ministère de l'éducation nationale, de la recherche et de la technologie (1999): L’organisation du système éducatif français, Paris.

MONTANDON C., PERRENOUD Ph. (1987): Entre parents et enseignants : un dialogue impossible?, Berne, Peter Lang.

OCDE (1997): Les parents partenaires de l'école, Paris, Centre pour la recherche et l'innovation dans l'enseignement.

PAYET J.-P. (1989): "L'action éducative entre école et famille», Annales de la recherche urbaine, 41.

PERRENOUD P. (1987) : «Le «go-between» : l'enfant messager et message entre sa famille et l'école» in MONTANDON C., PERRENOUD Ph., 1987

TAUVEL J.-P. (dir.) (1998): Les familles et l'école : une relation difficile, Paris, Ville-École-Intégration, Migrants-formation $\mathrm{n}^{\circ} 114$.

VAN ZANTEN A. (1998): "Les transformations des pratiques et des éthiques professionnelles dans les établissements d'enseignement» in Bourdoncle R., Demailly L. (coord) Les professions de l'éducation et de la formation, Lille, Presses du Septentrion. 